\title{
A multicentre study on admission hypothermia in very low-birth weight preterm infants in China: distribution, causes and risk factors
}

Yong-hui Yu ( $\sim$ alice20402@126.com )

Shandong Provincial Hospital https://orcid.org/0000-0001-7699-7482

\section{Li Wang}

Shandong Provincial Hospital

\section{Xiao-yu Dong}

Shandong Provincial Hospital

\section{Wen Li}

Shandong University Qilu Hospital

\section{Lei Huang}

Shandong provincal maternity and child health care hospital

\section{Li-ling Wang}

Shandong Qianfoshan Hospital

\section{Xiu-fang Fan}

jinan maternity and child health care hospital

\section{Yan-qiu Wu}

Qindao University Medical College Affiliated Yantai Yuhuangding Hospital

Chun-lei Zhang

weifang maternity and child health care hospital

\section{Xue-mei Sun}

Linyi People's Hospital

\section{Min Li}

linyi women's and children's hospital

\section{Yong-feng Zhang}

affiliated hospital of weifang medical college

\section{Guo Yao}

\section{Taian City Central Hospital}

\section{Cong Li}

Liaocheng People's Hospital

\section{Guo-ying Zhao}

binzhou medical university hospital

\section{Xin-feng Zhao}


zaozhuang maternity and child health care hospital

\section{Zhen-ying Yang}

taian maternity and child health care hospital

\section{Tong Chen}

dongying people's hospital

\section{Xue-yun Ren}

affiliated hospital of jining medical college

\section{Jing Li}

the second affiliated hospital of shandong first medical university

\section{Bing-ping Qiu}

tengzhou centarl hospital

\section{Shi-ping Niu}

zibo maternity and child health care hospital

\section{Ren-xia Zhu}

people's hospital of linzi district,zibo

\section{Yao Chen}

centarl hospital of shandong provincial affiliated to shandong university

\section{Yan-ling Gao}

dezhou people's hospital

\section{Ri-ming Zhao}

ju country people's hospital

\section{Li-ping Deng}

Heze Municipal Hospital

\section{Fu-dong Peng}

liaocheng second people's hospital

\section{Mei-rong Bi}

Jinan Central Hospital Affiliated to Shandong University

\section{Research article}

Keywords: Hypothermia, very low-birth weight infants, NICU, Shandong China

Posted Date: October 14th, 2019

DOI: https://doi.org/10.21203/rs.2.15988/v1

License: (c) (i) This work is licensed under a Creative Commons Attribution 4.0 International License. Read Full License 


\section{Abstract}

Background Neonatal hypothermia, defined as a temperature $<36.5^{\circ} \mathrm{C}$, is a major contributor to neonatal mortality and morbidity. Hypothermia in preterm infants remains a challenge in the neonatal intensive care unit (NICU) for many reasons.

Objective To investigate the incidence of admission hypothermia (AH) in very low-birth weight (VLBW) infants in multiple NICUs in Shandong Province, China, and to provide clinical evidence for the implementation of quality improvement practices to reduce the incidence of $\mathrm{AH}$ in NICU.

Methods This retrospective, observational study was carried out over a period of 12 months, from January 1, 2018 to December 31, 2018. The correlations between hypothermia preventive measures and the incidence of $\mathrm{AH}$ were analysed by descriptive statistical methods and a Spearman analysis. The associations between $\mathrm{AH}$ and maternal and neonatal variables were tested with bivariate analyses, followed by stepwise logistic regression. $\mathrm{P}<0.05$ was considered statistically significant.

Results A total of 1639 in-born infants who were born at a weight less than $1500 \mathrm{~g}$ were enrolled in the study on their day of birth. Among them, 1295 VLBW infants fulfilled the inclusion criteria. The incidence of $\mathrm{AH}$ was $87.9 \%$ in VLBW infants among the 28 NICUs. We investigated whether team training and education, such as monthly chart reporting on hypothermia in preterm infants on admission to the NICU, which was associated with $10.7 \%$ of all the cases, could decrease the rate of $\mathrm{AH}$. Indeed, transport with a heated transport incubator was associated with $9.3 \%$ of the cases. here was a negative correlation between the incidence of $\mathrm{AH}$ and the number of quality improvement measures implemented to prevent hypothermia $(r=-0.242, p<0.05)$. A low birth weight, intubation in the delivery room, and a low 5 -min Apgar score were significantly associated with $\mathrm{AH}$.

Conclusion: The incidence of $\mathrm{AH}$ was high. There was a negative correlation between the incidence of $\mathrm{AH}$ and the number of quality improvement measures implemented to prevent hypothermia. It is important to monitor for $\mathrm{AH}$ in newborns who have a low birth weight, who have a low Apgar score at $5 \mathrm{~min}$ and who require intubation in the delivery room.

\section{Background}

Because of the immature physiological development of preterm infants, they are susceptible to heat loss, potentially leading to hypothermia, especially very low-birth weight (VLBW) infants and extremely lowbirth weight (ELBW) infants [1]. The incidence of hypothermia on admission to the neonatal intensive care unit (NICU) in VLBW preterm infants is $31 \%-78 \%[2,3]$. Admission hypothermia (AH) is a vital risk factor for neonatal mortality and morbidity inpreterm infants $[4,5]$.

Maintaining normothermia in infants has been shown to improve survival and outcomes [6, 7]. In a multicentre study, Caldas et al. [8] reported that $\mathrm{AH}$ was significantly associated with early neonatal death regardless of hospital performance. In Korea, Lee et al. [9] reported that $74.1 \%$ of 5860 VLBW preterm 
infants ( $<33$ weeks of gestational age) with $\mathrm{AH}$ were admitted to neonatal units, and $\mathrm{AH}$ was associated with high mortality and several notable morbidities. Wilson et al. [10] reported that hypothermia occurred in $53.4 \%$ of 5697 infants born at $<32$ weeks of gestational age in a population-based study with samples from 11 European countries, and $\mathrm{AH}$ after very preterm birth was a significant factor associated with an increased risk of early and late neonatal mortality. Lyu et al. [11] showed that the rates of the composite outcome, severe neurological injury, severe retinopathy of prematurity (ROP), necrotizing enterocolitis (NEC), bronchopulmonary dysplasia (BPD), and nosocomial infection had U-shaped relationships with admission temperature, and the lowest rates of adverse outcomes were associated with admission temperatures between $36.5^{\circ} \mathrm{C}$ and $37.2^{\circ} \mathrm{C}$.

To reduce the rates of these complications, many clinical studies have suggested various measures to prevent hypothermia, including plastic wrapping without drying [12, 13, 14], the use of incubators [15], and the use of chemical mattresses [16]. Despite the knowledge and development of new approaches to better maintain infant body temperatures, recent studies have noted that a significant proportion of VLBW infants in undeveloped countries are not kept sufficiently warm immediately after birth [10, 17].

The aim of this study was to investigate the distribution, causes and risk factors of AH in VLBW infants in multiple NICUs in China.

\section{Methods}

\section{Study design and time period}

This retrospective, observational study was carried out over a period of 12 months, from January 1, 2018, to December 31, 2018, to investigate the incidence of AH in VLBW infants in multiple NICUs in Shandong Province, China.

\section{Study setting}

Shandong is located on the western shore of the Pacific; it has 3345 kilometres of coastline and borders on the Bohai Sea and the Yellow River, and its 158,000 square kilometres of landmass is home to over 100 million people. The study was conducted in twenty-eight public hospitals in Shandong, China, that have their own NICU. East Hospital of Shandong Provincial Hospital Affiliated to Shandong University has its own NICU, with an average NICU admission rate of 75 neonates per month. Qilu Hospital of Shandong University has an average NICU admission rate of 121 neonates per month. Shandong Provincial Maternity and Child Health Care Hospital has an average NICU admission rate of 83 neonates per month. Qianfo Shan Hospital Affiliated to Shandong University has an average NICU admission rate of 79 neonates per month. Jinan Maternity and Child Health Care Hospital has an average NICU admission of 180 neonates per month. Yantai Yuhuangding Hospital has an average NICU admission rate of 162 neonates per month. Weifang Maternity and Child Health Care Hospital has an average NICU admission rate of 326 neonates per month. Linyi People's Hospital has an average NICU admission rate of 349 
neonates per month. Linyi Women's and Children's Hospital has an average NICU admission rate of 370 neonates per month. The Affiliated Hospital of Weifang Medical College has an average NICU admission rate of 56 neonates per month. Taian Central Hospital has an average NICU admission rate of 171 neonates per month. Liaocheng People's Hospital has an average NICU admission rate of 253 neonates per month. Binzhou Medical University Hospital has an average NICU admission rate of 193 neonates per month. Zaozhuang Maternity and Child Health Care Hospital has an average NICU admission rate of 185 neonates per month. Taian Maternity and Child Health Care Hospital has an average NICU admission rate of 153 neonates per month. Dongying People's Hospital has an average NICU admission rate of 202 neonates per month. Affiliated Hospital of Jining Medical College has an average NICU admission rate of 530 neonates per month. The Second Affiliated Hospital of Shandong First Medical University has an average NICU admission rate of 100 neonates per month. Jinan Second Maternity and Child Health Care Hospital has an average NICU admission rate of 96 neonates per month. Tengzhou Central Hospital has an average NICU admission rate of 177 neonates per month. Zibo Maternity and Child Health Care Hospital has an average NICU admission rate of 161 neonates per month. The People's Hospital of Linzi District, Zibo, has an average NICU admission rate of 56 neonates per month. Central Hospital of Shandong Provincial Affiliated to Shandong University has an average NICU admission rate of 68 neonates per month. Ju County People's Hospital has an average NICU admission rate of 109 neonates per month. Heze Municipal Hospital has an average NICU admission rate of 51 neonates per month. Liaocheng Second People's Hospital has an average NICU admission rate of 72 neonates per month. Jinan Central Hospital has an average NICU admission rate of 49 neonates per month.

\section{Data measurement, data collection and data quality control procedures}

The admission temperature was defined as the infant's axillary or rectal temperature measured on admission to the NICU within one hour after birth, in accordance with local routines. Since January 1 , 2018 , homogeneous neonatal cooperative research platforms have been established. The admission temperatures, mortality incidence and morbidity data of VLBW infants born in 28 level-III NICUs in Shandong Province were collected prospectively. The database provided maternal, delivery, and neonatal data until the first NICU discharge, and the data were collected by trained staff using a standardized operating procedure [18]. The entered data were analysed for statistical adjustment of possible confounders with a multivariate analysis.

\section{Population}

\section{Study population}

The study population included all VLBW infants who were admitted to the NICUs of 24 level-III hospitals in Shandong Province, China, from January 1, 2018, to December 31, 2018, and their mothers. 


\section{Exclusion criteria}

VLBW infants who were out-born, families who rejected treatment, and infants with missing temperature data were excluded.

\section{Study variables}

\section{Dependent variable}

The dependent variable was $\mathrm{AH}$.

\section{Independent variables}

The following obstetric and neonatal variables were considered independent variables: diabetes, maternal hypertension, premature rupture of the membranes (PROM) ( $>24$ hours), and caesarean section. The following neonatal variables were considered independent variables: multiple birth (twins or more), sex, gestational age (GA), birth weight (BW), small for gestational age (SGA) (defined as growth below the 10th percentile), Apgar scores at $1 \mathrm{~min}$ and $5 \mathrm{~min}$, and intubation in the delivery room. Data on the implementation of measures to prevent hypothermia were collected using a retrospective questionnaire. The measures to prevent hypothermia were based on a review of the medical literature [19], best practice recommendations from the California Perinatal Quality Care Collaborative (CPQCC) [20], evidence-based principles from the Neonatal Resuscitation Program [21, 22], and recommendations by the World Health Organization (WHO) on ambient air temperatures in the delivery room [23]. The implementation of the measures to prevent hypothermia are described in Table 1.

\section{Operational definitions}

Hypothermia: an axillary temperature of less than $36.5^{\circ} \mathrm{C}$, as defined by the WHO [23]: cold stress or mild hypothermia, $36.0^{\circ} \mathrm{C}$ to $36.4^{\circ} \mathrm{C}$; moderate hypothermia, $32.0^{\circ} \mathrm{C}$ to $35.9^{\circ} \mathrm{C}$; and severe hypothermia, below $32^{\circ} \mathrm{C}$.

Normothermic: a body temperature of $36.5^{\circ} \mathrm{C}$ to $37.5^{\circ} \mathrm{C}$.

Out-born: the newborn was delivered at a site other than the study hospital.

\section{Statistical analysis}

Demographic data are expressed as means ( \pm standard deviations (SDs)) or percentages. The correlation between the hypothermia preventive measures and the incidence of $\mathrm{AH}$ was analysed by descriptive 
statistical methods and Spearman analysis. Associations between perinatal variables and hypothermia were tested with a bivariate analysis, followed by stepwise logistic regression. Separate models were constructed for combined moderate/severe and mild hypothermia. We also studied the potential contribution of hypothermia to the risk factors. Separate models were constructed for risk factors in which severe/moderate or mild hypothermia was the independent variable. $P<0.05$ was considered statistically significant. The statistical analyses were conducted using SPSS v. 25.0 (SPSS Inc., Chicago, Illinois).

\section{Results}

The 28 recruited hospitals included 20 general hospitals and 8 maternal and child health care hospitals, with an average of 59 and 40 beds in the neonatology departments and NICUs, respectively. A total of 1639 in-born infants born at a weight less than $1500 \mathrm{~g}$ were enrolled in the study on their day of birth; 97 infants were excluded because they were out-born. Additionally, 135 infants with families who ceased treatment and 94 infants who had missing temperature data were excluded. The remaining 1295 infants were included in this analysis (Fig. 1). The final cohort had a mean ( \pm SD) BW and GA of $1225 \pm 205 \mathrm{~g}$ and $29.7 \pm 2.1$ weeks, respectively.

\section{Hypothermia}

The mean (SD) admission temperature was $35.8^{\circ} \mathrm{C}\left(0.6^{\circ} \mathrm{C}\right)$, with a range of $32^{\circ} \mathrm{C}$ to $37.5^{\circ} \mathrm{C}$. Only $12 \%$ of the study population had an admission temperature in the WHO recommended range of $36.5^{\circ} \mathrm{C}$ to $37.5^{\circ} \mathrm{C}$. A total of $87.9 \%$ of infants had an admission temperature lower than $36.5^{\circ} \mathrm{C}$, including 567 infants (43.8\%) in the mild hypothermia group and 572 infants (44.2\%) in the moderate/severe hypothermia group. No hyperthermia $\left(>37.5^{\circ} \mathrm{C}\right)$ infants were identified. he distributions of infants across the range of admission temperatures according to BW are reported in Fig. 2.

\section{Cause of hypothermia}

Quality improvement methodologies such as Pareto charts were used to identify and prioritize the contributing reasons for $\mathrm{AH}$ (Fig. 3). We investigated whether team training and education could decrease the rate of $\mathrm{AH}$, including monthly chart reporting on hypothermia in preterm infants on admission to the NICU, which was associated with $10.7 \%$ of all the cases. Indeed, transport with a heated transport incubator was associated with $9.3 \%$ of the cases. In addition, $8.8 \%$ of the cases of hypothermia were associated with no measured and recorded body temperature $10 \mathrm{~min}$ after birth. There was a negative correlation between the incidence of $\mathrm{AH}$ and the number of quality improvement measures implemented to prevent hypothermia $(r=-0.227, p<0.05)$. (Fig. 4).

\section{Risk factors for hypothermia}


The univariate analysis of the risk factors of severe/moderate hypothermia indicated that BW, caesarean section, low 5-min Apgar scores, intubation in the delivery room, maternal hypertension, and SGA were associated with $\mathrm{AH}$ (Table 2). After adjusting for risk factors using a logistic regression analysis, $\mathrm{BW}$ [adjusted $O R 0.999,95 \% \mathrm{Cl}(0.998-1.000) ; p<0.05$ ], intubation in the delivery room [adjusted $O R 1.982$, $95 \% \mathrm{Cl}(1.078-3.318) ; p<0.05$ ], and a low 5-min Apgar score [adjusted $O R 2.159,95 \% \mathrm{Cl}(1.071-4.352)$; $p<0.05]$ remained significantly associated with moderate/severe hypothermia.

\section{Discussion}

This is the first large national cohort study to investigate the incidence of $\mathrm{AH}$ and its association with inhospital mortality and morbidity in the eastern region of China. Neonates regulate their body temperature much less efficiently than adults, and both hypothermia and hyperthermia can easily occur. Despite measures that were established in 2010 according to the recommendations of the Neonatal Resuscitation Program guidelines [21] to maintain normothermia in the delivery room, the incidence of AH is still high in China, occurring in $87.9 \%$ of VLBW infants. Mank et al. [24] reported that in $93 \%$ of infants, hypothermia occurred within the first three hours after admission. The proportion of infants with normal temperature ranges on NICU admission (12.0\%) is much lower in China than in other developed countries, such as US infants less than 29 weeks (52.9\%) [25], Canadian infants less than 33 weeks (57.2\%) [11] and European infants less than 32 weeks (42.2\%) [10].

The most notable infant characteristics that were associated with hypothermia were a low BW, intubation in the delivery room and a low Apgar score at $5 \mathrm{~min}$. A low BW is associated with a large surface area-tobody mass ratio, a low amount of subcutaneous fat, an increased body water content, immature skin leading to increased evaporative water and heat losses, a poorly developed metabolic mechanism for responding to thermal stress and the delayed development of skin blood-flow control, reducing the ability to maintain heat by vasoconstriction $[3,4,5]$, all of which may lead to hypothermia. Similar to our study, Laptook et al. [26] reported associations between BW and intubation in the delivery room and hypothermia. In addition, Lee et al. [9] reported that a low Apgar score at 5 min was a risk factor for hypothermia at admission. This may be related to the inadequate implementation of thermal protection measures during resuscitation.

The results of the correlation analysis indicated that the incidence of $\mathrm{AH}$ was effectively reduced with an increased number of hypothermia prevention measures, as there was a negative correlation between the incidence of $\mathrm{AH}$ and the number of implemented intervention measures. A retrospective study from the Vermont Oxford Network's (VON) VLBW database showed that the use of chemical warming packs in addition to routine care may be a useful intervention in achieving normothermia during the transition from delivery to admission to the NICU [27]. Recent studies reported that the use of plastic wrapping without drying reduced the rate of $A H[12,13,14]$. Wilson et al. [28] investigated the different strategies used in 11 European countries to prevent hypothermia. The results of the study showed that very preterm infants had a reduced risk of hypothermia at NICU admission if the unit used systematic prevention 
strategies and that all the strategies had similar effects, possibly due to implementation rather than a strategy's specific efficacy.

In addition, a retrospective analysis of the practices to prevent hypothermia revealed that team training and education, including monthly chart reporting on hypothermia in preterm infants on admission to the $\mathrm{NICU}$ and transportation with a heated transport incubator, were key drivers of change. Although special space for the implementation of these interventions is not required, the reluctance to implement such measures may be associated with poor awareness by medical staff, a lack of infrastructure or funding, and so on. Based on these findings, implementing interventions to prevent hypothermia and enhancing team building were identified as key drivers of change. Based on recent studies in developed countries, after initiating quality improvement projects [29, 30,31,32], the incidence of AH dramatically declined. Thus, we need to implement evidence-based practices for improving quality (EPIQ) to reduce the incidence of $\mathrm{AH}$ in VLBW infants.

Our study had several limitations. We investigated only the incidence of $\mathrm{AH}$ and studied the association between $\mathrm{AH}$ and risk factors; we could not conduct a quality improvement project considering VLBW infants. Based on the results of this study, our next research project will be to carry out a multicentre quality improvement project to reduce the incidence of $\mathrm{AH}$ according to international EPIQ.

\section{Conclusions}

The incidence of $\mathrm{AH}$ was high. There was a negative correlation between the incidence of $\mathrm{AH}$ and the number of quality improvement measures implemented to prevent hypothermia. It is important to monitor for $\mathrm{AH}$ in newborns who have a low BW, who have a low Apgar score at $5 \mathrm{~min}$ and who require intubation in the delivery room.

\section{Abbreviations}

VLBW, very low-birth weight; NICU, neonatal intensive care unit; AH, admission hypothermia; OR, odds ratio; $\mathrm{Cl}$, confidence interval; SGA, small for gestational age; PROM, premature rupture of the membranes.

\section{Declarations}

\section{Acknowledgements}

We would like to thank Yuan Shi, Professor, from the Chongqing Children's Hospital and Zhang-bin Yu from the Nanjing Maternal and Child Health Hospital of Nanjing Medical University for the assistance with this research project.

\section{Funding}


The Shandong Key Research and Development Project (2018GSF118163) and Shandong Provincial Medical Health Technology Development Project (2017WS009) had covered all the costs for data collection instruments, data collection, data entry and payments for supervisors and advisors.

\section{Availability of data and materials}

The data that support the findings of this study are available from the corresponding authors upon reasonable request.

\section{Ethics approval and consent to participate}

Ethical approval with ethics approval number of LCYJ: NO. 2019-004 was obtained

from the Institutional Review Board of Shandong Provincial Hospital Affiliated with Shandong University. In our study, verbal informed consent was obstained from all participants. The Institutional Review Board of Shandong Provincial Hospital Affiliated with Shandong University considered the project exempt from written informed consent because this study was retrospective and observational study.

\section{Consent for publication}

Not applicable.

\section{Authors' contributions}

$\mathrm{YYH}$, the corresponding author, designed the study, trained and supervised the data collectors, interpreted the results and revised the manuscript. The first authors, namely, WL, played a role in the analysis and interpretation of the data and in preparing and drafting the manuscript.The co-first authors, namely, LW, HL, WLL, FXF, WFQ, ZCL, SXM, LM, ZYF,YG,LC, ZGY, ZXF, YZYZ, CT, RXY, LJ, QBP, NSP, ZRX, CY, GYL, ZRM, DLP, PFD, and BMR, participated in the design of the study, the collection and interpretation of the data and writing the manuscript. All authors listed on the manuscript approved the submission of this version of the manuscript and take full responsibility for the manuscript. The second authors, namely, DXY, played a role in the collection of the data.

\section{Competing interests}

The authors declare that they have no conflicts of interest.

\section{References}


1. Bhatt DR, White R, Martin G. Transitional hypothermia in preterm newborns. Perinatol. 2007; 27: S45S47.

2. Boo NY, Guat-Sim Cheah I, Malaysian National Neonatal Registry. Admission Hypothermia among VLBW Infants in Malaysian NICUs. J Trop Pediatr. 2013; 59(6): 447-452.

3. Aylott M. The neonatal energy triangle. Part2: thermoregulatory and respiratory adaption. Paediatr Nurs. 2006; 18: 38-42.

4. Hammarlund K, Sedin G. Transepidermal water loss in newborn infants. VI. Heat exchange with the environment in relation to gestational age. Acta Paediatr Scand. 1982; 71: 191-196.

5. Costarino A, Baumgart S. Modern fluid and electrolyte management of the critically ill premature infant. Pediatr Clin North Am. 1986; 33: 153-178.

6. Silverman WA, Balnc WA. The effect of humidity on survival of newly born premature infants. Pediatrics. 1957; 20: 477-486.

7. Buetow KC, Klein SW. Effect of maintenance of 'normal' skin temperature on survival of infants of low birth weight. Pediatrics. 1964; 34: 163-170.

8. Caldas JP, Ferri WAG, Marba STM. Admission hypothermia, neonatal morbidity, and mortality: evaluation of a multicenter cohort of very low birth weight preterm infants according to relative performance of the center. Eur J Pediatr. 2019; 5: 6.

9. Lee NH, Nam SK, Lee J. Clinical impact of admission hypothermia in very low birth weight infants: Results from Korean neonatal network. Korean J Pediatr. 2019; 5: 22.

10. Wilson E, Maier RF, Effective Perinatal Intensive Care in Europe(EPICE) Research Group.Admission hypothermia in very preterm infants and neonatal mortality and morbidity. J Pediatr, 2016; 175: 6167. e4.

11. Lyu Y, Shah PS, Ye XY. Association between admission temperature and mortality and major morbidity in preterm infants born at fewer than 33 weeks' gestation. JAMA Pediatr. 2015; 169: e150277.

12. Leadford A, Warren J, Manasyan A. Plastic bags for prevention of hypothermia in preterm and low birth weight infants. Pediatrics, 2013; 132: e128-e134.

13. Smith J, Usher K, Alcock G. Application of plastic wrap to improve temperatures in infants born less than 30 weeks gestation: a randomized controlled trial. Neonatal Netw. 2013; 32: 235-245.

14. Liu Yanli, Wei Zhou, Tang Zhenhai, Chen Si, Lin Zhenlang. Effectiveness of plastic wrap in prevention of hypothermia in very low birth weight infants. Chin J Perinat Med. 2014; 17(4): 244-248.

15. Chawla S, Amaram A, Gopal SP, Natarajan G. Safety and effcacy of Transwarmer mattress for preterm neonates: results of a randomized controlled trial. Perinatol. 2011; 31: 780-784.

16. Ibrahim, Yoxall. Use of self-heating gel mattresses eliminates admission hypothermia in infants born below 28 weeks gestation.Eur J Pediatr. 2010; 169(7): 795-799.

17. Ukke GG, Diriba K, Prevalence and factors associated with neonatal hypothermia on admission to neonatal intensive care units in Southwest Ethiopia-A cross-sectional study. PLoS ONE. 2019; 14(6): 
e0218020.

18. Li Wang, Yong-hui Yu, Shandong Multicenter Study Coordination for Admission Hypothermia in Neonatal Intensive Care Units. Evidence-based practice for improving quality to to reduce the incidence of admission hypothermia: a multicentered study protocol. Chin J Based Pediatr. 2019; 14(2): 121-123.

19. Fawcett K. Preventing Admission Hypothermia in Very Low Birth Weight Neonates. Neonatal Netw, $2014 ; 33(3):$ 143-149.

20. Bell R, Finer N, Halamek L. Delivery room management: quality improvement toolkit [monograph on the Internet]. Stanford, CA: California Perinatal Quality Care Collaborative; 2011, Jul.

21. Kattwinkel J, Perlman JM, Aziz K. American Heart Association. Neonatal resuscitation: 2010 American Heart Association Guidelines for Cardiopulmonary Resuscitation and Emergency Cardiovascular Care. Pediatrics. 2010; 126(5): e1400-e1413.

22. Hong-mao Ye, Chinese Neonatal Resuscitation Project. Neonatal Resuscitation Program guidelines in China (Beijing revision in 2016). Chin J Perinat Med, 2016; 19(7): 481-486.

23. World Health Organization. Department of Reproductive Health Research. Thermal protection of the newborn: a practical guide. http://whqlibdoc.who.int/hq/1997/WHO_RHT_MSM_97.2.pdf. Published 1997.

24. Mank A, van Zanten HA, Meyer MP. Hypothermia in Preterm Infants in the First Hours after Birth: Occurrence, Course and Risk Factors. PLoS ONE, 2016; 11(11): e0164817.

25. Laptook AR, Bell EF, Seetha Shankaran. Neonatal Research Network. Admission Temperature and Associated Mortality and Morbidity among Moderately and Extremely Preterm Infants. J Pediatrics. 2018; 192: 53-59. e2.

26. Laptook AR, Salhab W, Bhaskar B. Neonatal Research Network. Admission temperature of low birth weight infants: predictors and associated morbidities. Pediatrics. 2007; 119(3): e643-e649.

27. Pinheiro JMB, Boynton S, Furdon SA. Use of chemical warming packs during delivery room resuscitation is associated with decreased rates of hypothermia in very low-birth-weight neonates. Adv Neonatal Care. 2011; 11: 357-362.

28. Wilson E, Zeitlin J, Piedvache A, Misselwitz B. Cohort study from 11 European countries highlighted differences in the use and efficacy of hypothermia prevention strategies after very preterm birth. Acta Paediatr. 2018; 107(6):958-966.

29. Caldas JP, Millen FC, Camargo JF. Effectiveness of a measure program to prevent admission hypothermia in very low-birth weight preterm infants. J Pediatr (Rio J). 2018, 94: 368-373.

30. Han Saem Choi, Soon Min Lee, Ran Namgung. The impact of a quality improvement effort in reducing admission hypothermia in preterm infants following delivery. Korean J Pediatr. 2018; 61(8): 239-244.

31. Andrews C, Whatley C, Smith M. Quality-Improvement Effort to Reduce Hypothermia Among HighRisk Infants on a Mother-Infant Unit. Pediatrics. 2018; 141(3): e20171214. 
32. Frazer M, Ciarlo A, Herr J, Briere CE. Quality Improvement Initiative to Prevent Admission Hypothermia in Very-Low-Birth-Weight Newborns. J Obstet Gynecol Neonatal Nurs. 2018; 47(4): 520528.

\section{Tables}

Table 1 Implementation measures to prevent hypothermia

\begin{tabular}{ll}
\hline $\begin{array}{l}\text { Numerical } \\
\text { order }\end{array}$ & Intervention \\
\hline 01 & $\begin{array}{l}\text { Predelivery preparation } \\
\text { Ambient temperature at } 25^{\circ} \mathrm{C} \text { and the resuscitation bed on manual control with maximum heat } \\
\text { output }\end{array}$ \\
02 & Pre-warmed hat and blanket prepared \\
03 & Polyethylene wrap prepared \\
& Birth \\
04 & Infant immediately wrapped with a polyethylene wrap without drying \\
05 & Infant wrapped in a pre-warmed blanket wrap after the umbilical cord is cut \\
06 & Infant quickly weighed after being placed in a pre-warmed blanket \\
07 & A pre-warmed hat is placed on the head \\
08 & Body temperature measurement and recording 10 min after birth \\
& Before and at NICU admission \\
09 & Infant transported with a heated transport incubator \\
10 & All surfaces that the infant will contact are pre-warmed \\
11 & Infant is immediately placed in the admission bed (e.g. Giraffe OmniBed) \\
12 & Infant's body temperature is measured within 1 hour after birth \\
13 & Infant receives hypothermic rewarming \\
14 & The time of admission temperature $\geq 36.5^{\circ} \mathrm{C}$ is recorded \\
& Team training and education \\
15 & Both nursing and medical operations implemented \\
16 & Monthly chart reporting on hypothermia in preterm infants on admission to the NICU \\
17 & Temperature measurement standardized \\
18 & Training and assessments on temperature measurement for nurses \\
& Implementing quality improvement projects to reduce hypothermia in preterm infants on \\
& admission to the NICU \\
\hline
\end{tabular}

Table 2 Risk factors for moderate/severe hypothermia 


\begin{tabular}{|c|c|c|c|c|}
\hline & $\begin{array}{l}\text { Moderate/severe } \\
\text { hypothermia (\%) }\end{array}$ & $\begin{array}{l}\text { Mild } \\
\text { hypothermia (\%) }\end{array}$ & $\begin{array}{l}\text { Normothermia } \\
(\%)\end{array}$ & $p$ \\
\hline GA, mean (SD), wk & $29.6(2.1)$ & $29.8(2.0)$ & $29.8(2.1)$ & 0.835 \\
\hline $\mathrm{BW}$, mean (SD), g & 1200 (219) & $1241(194)$ & $1270 \square 180 \square$ & 0.004 \\
\hline SGA & $162(28.3)$ & 113 (19.9) & $28(17.9)$ & 0.001 \\
\hline Sex (boy) & $297(51.9)$ & $286(50.4)$ & $84(53.8)$ & 0.664 \\
\hline Caesarean section & $435(76.0)$ & $410(72.3)$ & $106(67.9)$ & 0.026 \\
\hline $\begin{array}{l}\text { Multiple birth (twins or } \\
\text { more) }\end{array}$ & 109 (19.1) & $113(19.9)$ & $25(16.0)$ & 0.547 \\
\hline $\begin{array}{l}\text { Apgar score at } 1 \\
\min <7\end{array}$ & $212(37.1)$ & $197(34.7)$ & $44(28.2)$ & 0.119 \\
\hline $\begin{array}{l}\text { Apgar score at } 5 \\
\min <7\end{array}$ & $100(17.5)$ & $68(12.0)$ & $11(7.1)$ & 0.001 \\
\hline $\begin{array}{l}\text { Intubation in the } \\
\text { delivery room }\end{array}$ & $146(25.5)$ & $110(19.4)$ & $19(12.2)$ & 0.001 \\
\hline Maternal hypertension & $259(45.3)$ & $225(39.7)$ & $49(31.4)$ & 0.005 \\
\hline Diabetes & $64(11.2)$ & $62(10.9)$ & $19(12.2)$ & 0.909 \\
\hline PROM & $147(25.7)$ & $169(29.8)$ & $40(25.6)$ & 0.258 \\
\hline
\end{tabular}

Abbreviations: SGA, small for gestational age; PROM, premature rupture of the membranes

Table 3 Risk factors for the WHO criteria of moderate/severe hypothermia and mild hypothermia as determined by logistic regression models 


\begin{tabular}{|c|c|c|c|}
\hline & \multicolumn{3}{|l|}{ Adjusted $O R^{*}(95 \% C I)$} \\
\hline & $\begin{array}{l}\text { Moderate/Severe } \\
\text { hypothermia }\end{array}$ & $\begin{array}{l}\text { Mild } \\
\text { hypothermia }\end{array}$ & Normothermia \\
\hline BW, mean (SD), g & $0.999(0.998-1.000)^{\mathrm{a}}$ & $\begin{array}{c}1.000(0.999- \\
1.001)\end{array}$ & 1 \\
\hline Caesarean section & $1.256(0.810-946)$ & $\begin{array}{l}1.111(0.725- \\
1.702)\end{array}$ & 1 \\
\hline Apgar score at $5 \min <7$ & $2.159(1.071-4.352)^{\mathrm{a}}$ & $\begin{array}{c}1.562(0.770- \\
3.169)\end{array}$ & 1 \\
\hline $\begin{array}{l}\text { Intubation in the delivery } \\
\text { room }\end{array}$ & $1.982(1.078-3.318)^{\mathrm{a}}$ & $\begin{array}{l}1.513(0.861- \\
2.657)\end{array}$ & 1 \\
\hline Maternal hypertension & $1.393(0.906-2.141)$ & $\begin{array}{l}1.349(0.881- \\
2.065)\end{array}$ & 1 \\
\hline SGA & $1.484(0.930-2.367)$ & $\begin{array}{c}0.917(0.572- \\
1.471)\end{array}$ & 1 \\
\hline
\end{tabular}

Abbreviations: OR, odds ratio; CI, confidence interval; SGA, small for gestational age; PROM, premature rupture of the membranes

*Adjusted for caesarean section, BW, SGA, Apgar score $<7$ at 5 min, and intubation in the delivery room.

$\mathrm{a}_{\text {odds ratios with } p<0.05}$

\section{Figures}




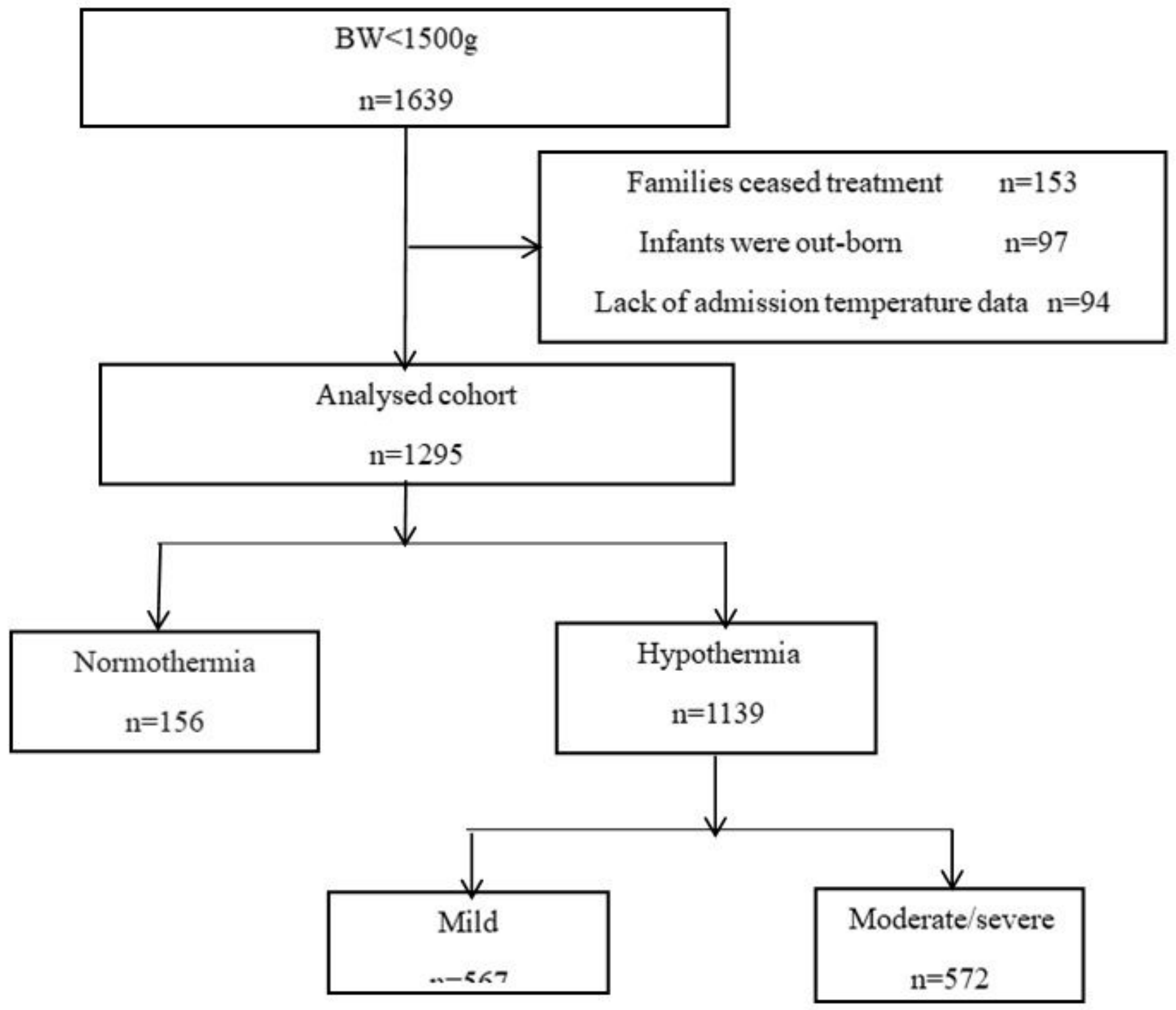

Figure 1

Flow diagram of the study population. 


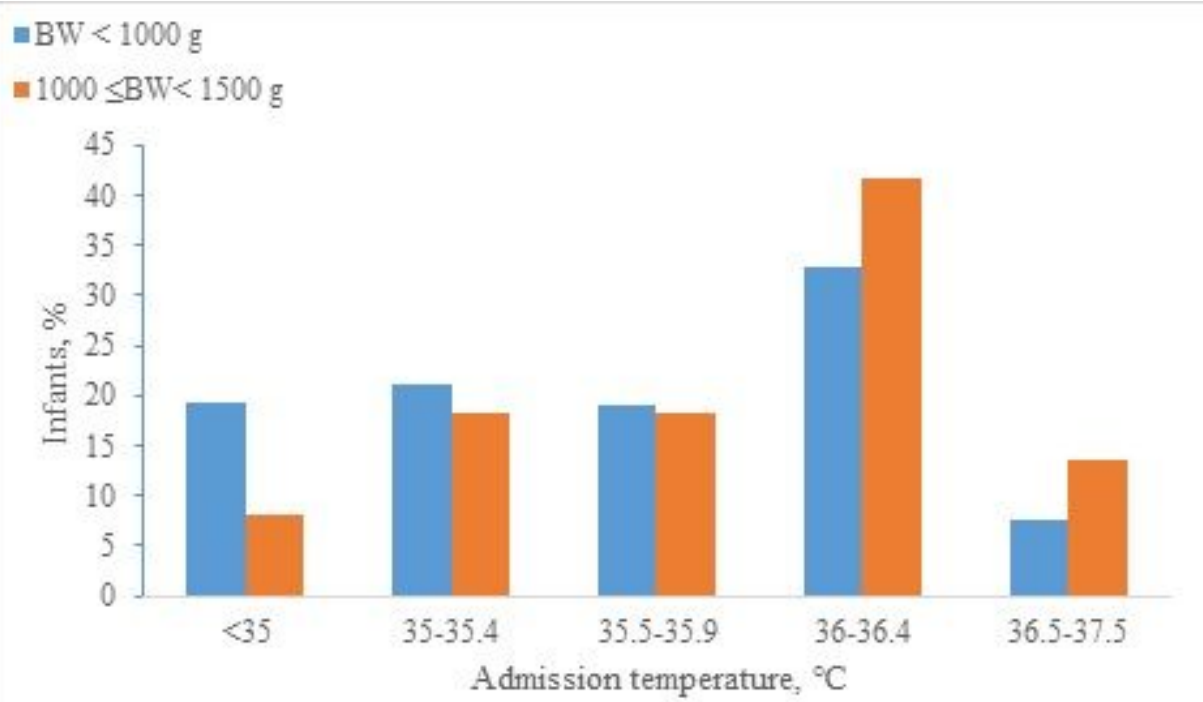

Figure 2

Temperature distribution according to birth weight.

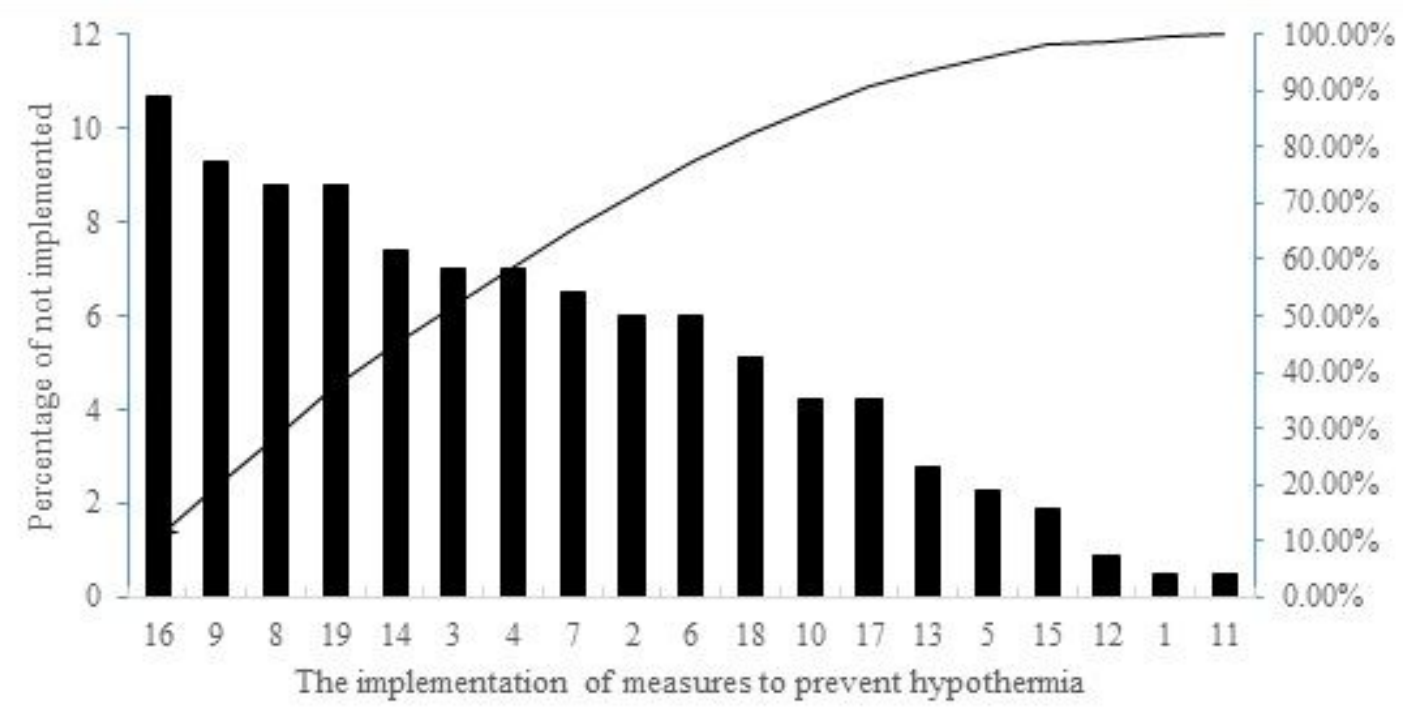

Figure 3

Pareto chart of the reasons for hypothermia. 


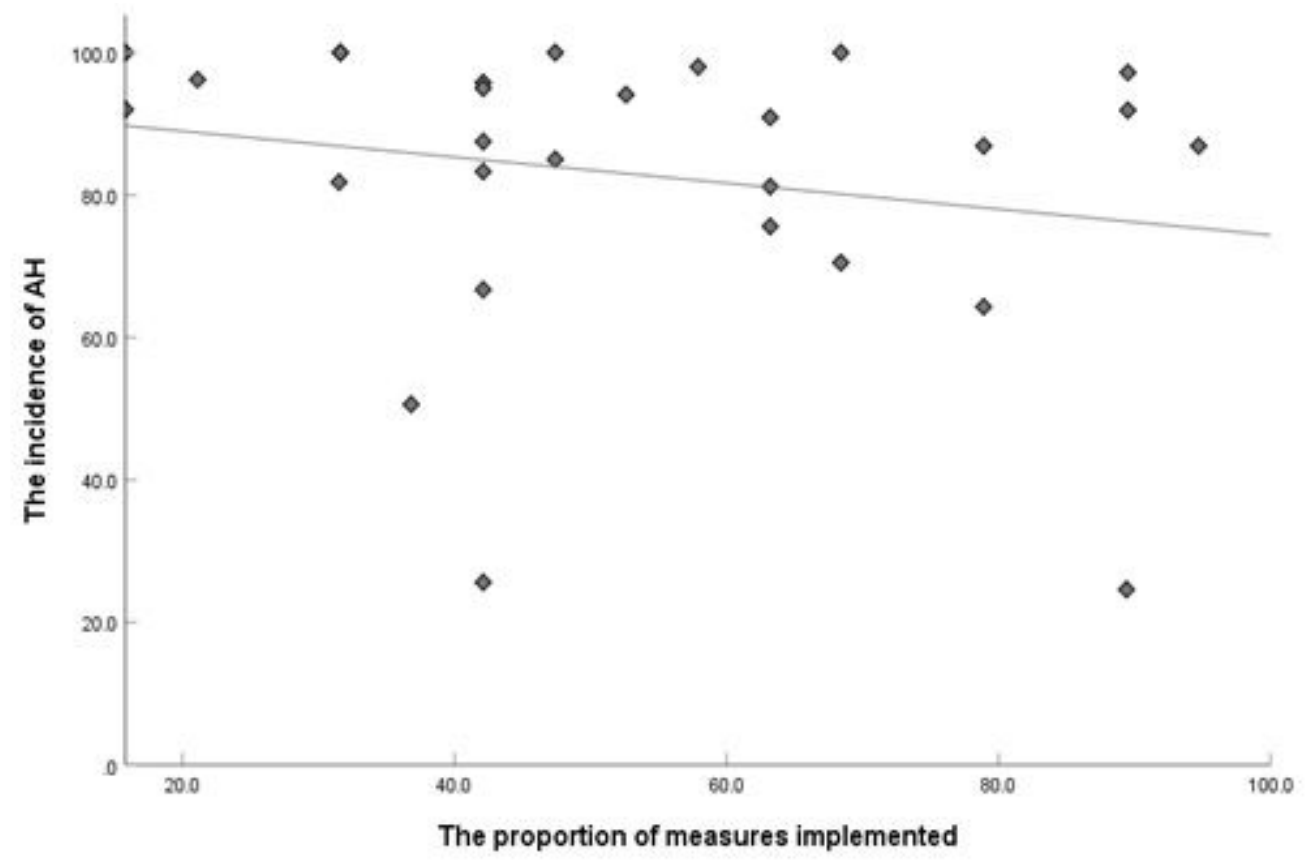

\section{Figure 4}

The correlation between the number of measures implemented and the incidence of $\mathrm{AH}$. 